\title{
A Video Service Cloud Platform with Multi-Network Convergence Application
}

\author{
Hong Liu \\ School of Computer Science, \\ Beijing University of Posts and Telecommunications, \\ Beijing, China \\ Gang Li \\ Beijing Peony Electronic Group Co., Ltd., \\ No.2 Huayuan Road, Haidian District, \\ Beijing, China
}

\author{
MinLu Dai \\ Beijing Peony Electronic Group Co., Ltd., \\ No.2 Huayuan Road, Haidian District, \\ Beijing, China \\ FangChun Yang \\ School of Computer Science, \\ Beijing University of Posts and Telecommunications, \\ Beijing, China
}

\section{OVERALL FRAMEWORK}

Abstract-With network technology advances, how to take advantages of different networks to provide users with creative and convergence service experience is of great strategic significance to tri-play service providers. A new video service cloud platform - iVideo platform is proposed in this paper. The overall framework and functional modules are presented, and the system workflow is also described. The iVideo platform provides user with intelligent cross-screen content cloud synchronization service on various devices, such as STB, PC, pad and mobile phone. It can enjoy user with downloadable high quality videos and also the cross-network convergence service experience. Moreover, it supports content protection with DRM deployment, which is beneficial to establishing a benign business environment.

Index Terms - Intelligent video service, Cloud platform, DRM, Multiple network, Cross-screen convergence

\section{INTRODUCTION}

With the development of network technology, the network infrastructure has obtained great development whether the cable television network, the internet, or the mobile communication network. At present, all three networks already have the ability to provide integrated services such as video, data and voice, in which the video service has greater add-values compared to the voice and data service. Thus, how to take advantages of triple networks to provide subscribers with convenient, flexible, and high quality video service is of great strategic significance to tri-play service providers.

This paper proposes a video service cloud platform to provide ntelligent cross-screen video service, bellow called iVideo platform. The iVideo has advantages of multi-screen access, multi-screen synchronization, multi-screen download, and multi-screen management. Unlike most other video systems, the iVideo platform supports digital copyright protection, that is, the protected video contents are encrypted and only authorized subscriber has the right to play the contents. This feature can be a good support of various content transaction business model such as free, time-limit, and payper-view video service.
The iVideo Platform works in muti-network environment, its overall framework consists of four systems: content system (CS), service operating and support system (SOSS), access and transmission network (ATN), and subscriber device (SD). The platform framework is shown in Fig.1.

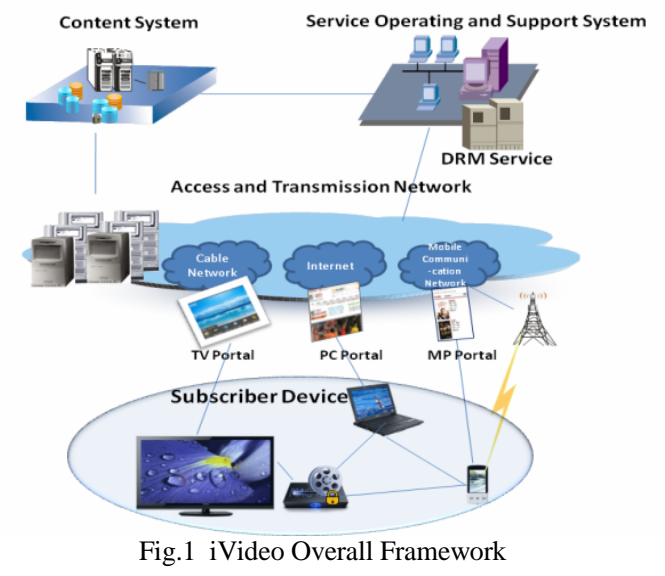

\section{A. Content System (CS)}

The CS consists of content capturer and encoder, encryption server, web store, and content server. The main functions are as follows: capture video and audio resources; encode and encrypt media files; store files and make content catalogue for search service; provide web store for subscriber to browse, search, purchase, and download content item interface for different kind of device, include TV, PC, or mobile phone; the contents are encrypted and only authorized subscribers can play the contents.

\section{B. Service operating and support system (SOSS)}

The SOSS is the core of the iVideo platform to provide converged service operating and support. The SOSS consists 
of DRM server, transaction server, payment server, registrar and authentication server, and service sync gateway. The DRM server is up to assign licenses according to users or user device. The transaction server treats the user's purchase procedure. The payment server provides various payment way. When the user pays for the content, it connects to the payment server. The service sync gateway controls all the service process, and is up to intelligent synchronize content among user devices, by sending content server URL to the target device, then the device can download the content media file from the content server. The register and authentication server manages user and user device register and activation, and maintains their binding relations.

The SOSS provide multi-device access and cross-screen service synchronization. For example, the subscriber can use his mobile phone to browse and purchase content, select STB in his home to download the content, and then play the content from STB. The contents that user purchased also can be synchronized to his all registered device by cloud platform.

The SOSS is based on secure architecture, and is open to be integrated with third-play payment and content provider.

\section{Access and transmission network (ATN)}

ATN provides network access service and content delivery service. Different kinds of devices access iVideo service via different networks. The STB is connected to CATV, PC and pad are connected to internet, and mobile phone is connected to mobile communication network. ATN ensures reliably delivery content to devices with a high rate.

\section{Subscriber device (SD)}

The iVideo platform provides services for various devices, include STB, PC, Pad and mobile phone. The iVideo client software is installed on devices. It communicates with SOSS server to register, activation or acquire licenses to play DRM protected content. As the client gets the license, it can decrypt the media file and play it according to the license.

The Fig.2 illustrates the iVideo platform function modules.
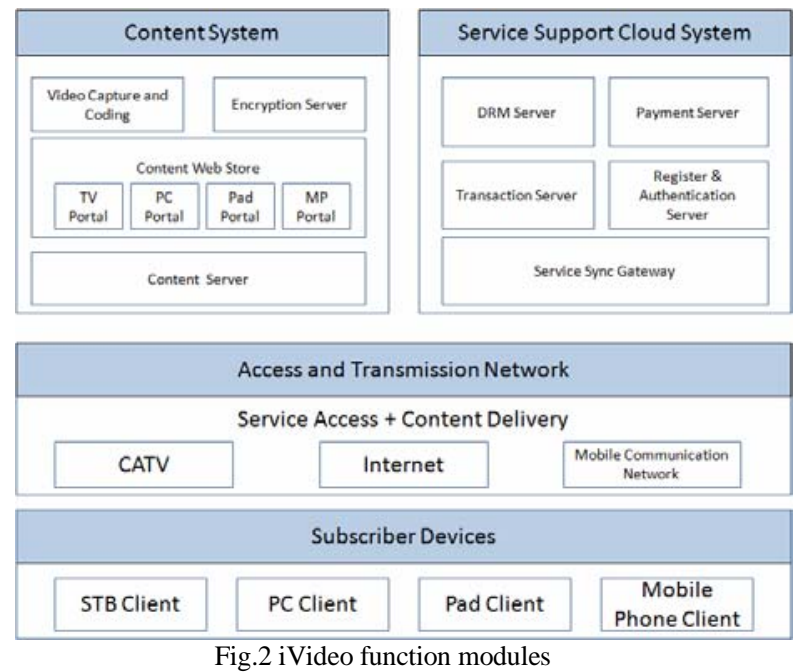

\section{MULTI-SCREEN VIDEO CLOUD SERVICE}

The iVideo platform provides multi-network cross-screen video service, supports automatic content cloud synchronization on user's devices, so users can enjoy video anytime and anywhere.

Following a typical user case is described as an example to explain the service model.

Tom usually uses five devices to watch video: TV with set top box (STB), PC at home, PC at office, mobile phone and pad. The STB connects to iVideo via CATV network with cable modem. The PC and pad connect to Internet via adsl, ethernet or wifi. The mobile phone connects to the $2 \mathrm{G}$ or $3 \mathrm{G}$ mobile communication network. The iVideo client has installed on these five devices.

On lunch café rest, Tom uses mobile phone to log onto the iVideo web store, browse and purchase a hot movie by mobile payment. When payment, the platform will inform Tom that he could watch the movie any times within 48 hours since he first plays the movie. Then the platform will list Tom's devices, and let him to select devices to download the movie. Tom wants to watch the movie on TV, so he selects his STB as the target device. After that, the iVideo SOSS will automatically push the movie Tom purchased to his STB through network. When Tom comes home from work, maybe the movie has finished download to his STB, so Tom can turn on TV to watch the movie with STB any time he want. When Tom plays the movie, the iVideo client installed on STB will automatically acquire license from SOSS DRM server, and Tom does not need to do any more work. At the same time, the iVideo client will control the play permissions according to the license, and remind Tom watch the movie during the valid time.

The Fig.3 illustrates the user case described above.

Similarly, Tom can use PC in office to purchase contents and designate PC in home as the download target device. The iVideo platform provides multi-device service synchronization and cross-screen service to push the contents to any devices the user designated.

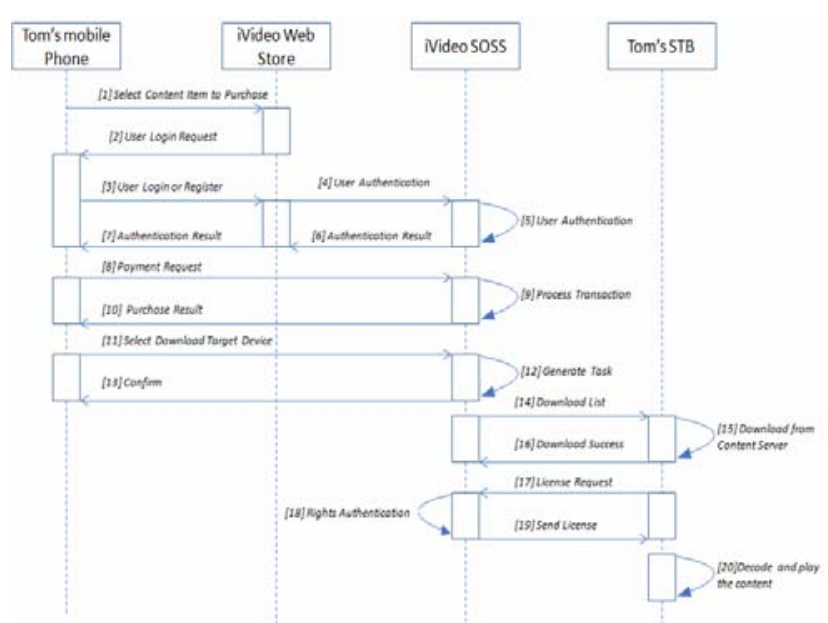

Fig.3 Service User Case 


\section{DRM SERVICE}

The iVideo platform provides content copyright protection with DRM technology, and support various business model such as pre-sale, pay per view, time-limited service. The DRM system is founded on internet secure technology include PKI technical architecture, digital encryption technology and digital signature, etc.

The DRM functional procedure includes subscriber register and device activation; authenticate subscriber and device data; generate digital license for authorized user; renew secure data; and feedback user's behavior.

The Fig.4 shows the functional block related to DRM services and their relationships.

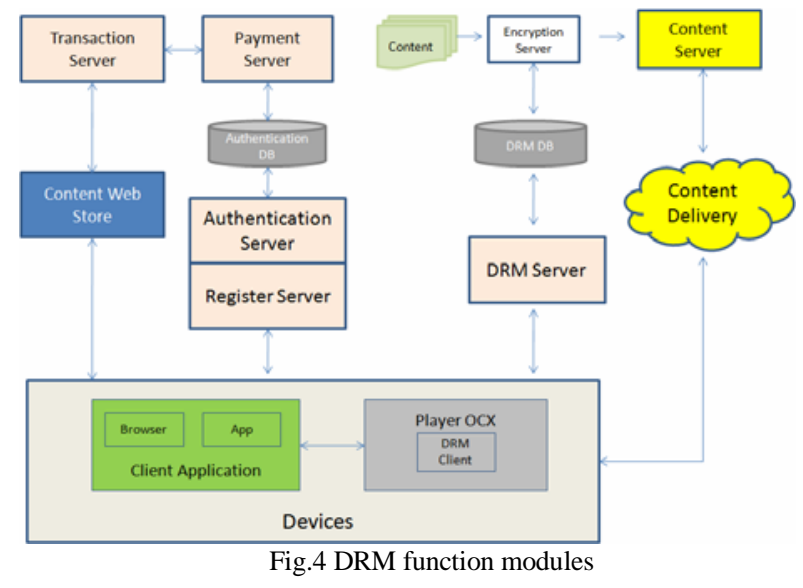

\section{SYSTEM WORK FLOW}

The Fig.5 describes the main system work flow. There are five main service processes: encryption; rights management; device activation; client login and acquire license; content delivery.

\section{A. Encryption}

Once media files are prepared, it is encrypted using a encryption tool which will create an entry with its associated cipher texts in the DRM DB. After encryption, the system publishes content to make it available at content server. The user's rights to view the content are controlled by payment server.

\section{B. Rights management}

Rights management is controlled by the payment server. DRM DB will keep track of the devices used to playback files. It will therefore enforce that any movie can't be played on more than the restricted number of devices allowed.

\section{Device activation}

A device has to register itself through the device registrar using the user's login name and password. When the device is activated, it downloads the files needed by DRM client. These include the systems' public key known as "be.PK”, and user's corresponding private key known as DK to the local store. For security reason, the private key (DK) will be encrypted before storing to the local disk. Copying the encrypted DK to other machine will not work.

\section{Client login and acquire license}

Once activated, the client player should be able to playback any DRM protected movies provided that the license allows. The client player goes through the following process:

- Web/UI login with its login name or saved credentials;

- $\quad$ Player asks DRM client to obtain license;

- License server(also called DRM server) consults payment server for users rights against the content;

- License server checks if playback does not exceed the maximum allowed;

- License server issues the resultant rights (either granted or denied could happen) to client.

\section{E. Content delivery}

The device client timing queries the SOSS to get synchronized content list and the content server URL. The content can be delivered either by using Peer to Peer technology or simply through HTTP server or FTP server.

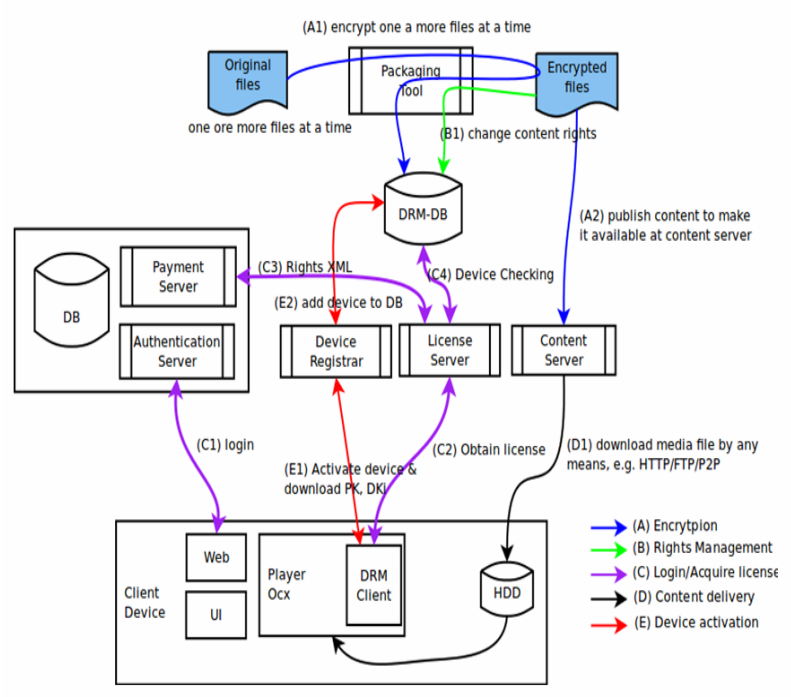

Fig.5 System work flow

\section{CONCLUSION}

This paper proposes a new video service platform iVideo, which provides multi-network converged video cloud services. The overall framework involves content system, service operating and support system (SOSS), access and transmission network, and subscriber device. The SOSS is the core of iVideo platform to provide multi-device access and content automatically cloud synchronization. The cross-screen download service is not network layer convergence, but really a service convergence, which brings not only the high quality video enjoy to user but also anytime and anywhere video experience. Unlike the most video web service, the iVideo 
supports content copyright protection with DRM technology, which is of benefit to establishing a benign business environment.

\section{REFERENCES}

[1] G. Wan, L. Li, "Broadcast Television Network Operator Broadband Business Development Strategy Preliminary of Triple Play,” China Digital Cable TV, pp.11-13, Jan. 2012.

[2] D.Z. Bao, "Struggles among New Businesses in the Integration of Three Network," China New Telecommunications, vol 21, pp.15-17, April 2010.
[3] S.William, "Network Security Essentials," Prentice Hall, ISBN0-13-120271-5, 2000.

[4] D.Y. Zhang, Y. F. He, P. Yan, W.Q. Shu, "Video Cloud Architecture in Three Network Convergence," Radio \& TV Broadcast Engineering, vol 38, pp. 42-47, May 2011.

[5] H.Z. Zhao, P.Yuan, L. C. Xie, "ID Control and Management Solution of Digital Rights Management in Tri-networks Intgration,” Computer Engineering, pp. 17-29, Nov. 2012.

[6] G. Richard, "Requirements for DRM Systems," Proc. Of Digital Rights Management, pp. 16-25, Jan. 2003. 